\title{
ISÓTOPOS ESTÁVEIS: FUNDAMENTOS E TÉCNICAS APLICADAS À CARACTERIZAÇÃO E PROVENIÊNCIA GEOGRÁFICA DE PRODUTOS ALIMENTÍCIOS.
}

\author{
Fabrício A. Caxito ${ }^{1}$, Almir Vieira Silva ${ }^{2}$ \\ 1- Centro de Pesquisa Manoel Teixeira da Costa - Departamento de Geologia - Universidade Federal de Minas Gerais; Av. Antônio Carlos 6627, \\ Pampulha, CEP 31270901, Belo Horizonte, MG, Brasil. boni@ufmg.br \\ 2- Universidade Federal do Oeste da Bahia - UFOB, Rua Professor Jose Seabra de Lemos, 316; Recanto dos Passaros, Barreiras, BA, CEP $47808-021$. \\ almir.silva@ufob.edu.br \\ Recebido em 1 de maio de 2015; aceito em 15 de julho de 2015
}

Resumo: Nos últimos anos, a utilização de isótopos estáveis como traçadores da proveniência geográfica de produtos alimentícios vem crescendo e a força desta ferramenta na determinação da origem de produtos com Denominação de Origem Controlada vem sendo demonstrada. Neste sentido, os isótopos de $\mathrm{C}, \mathrm{O}, \mathrm{N}, \mathrm{H}, \mathrm{S}$, e Sr mostram-se especialmente úteis. Neste artigo, os fundamentos do método e suas principais técnicas são revistos, de forma a fornecer as bases para a compreensão e interpretação destes dados.

Palavras Chave: Isótopos estáveis, proveniência geográfica, Denominação de Origem Controlada

Abstract: STABLE ISOTOPES: FUNDAMENTALS AND APPLIED TECHNIQUES TO THE CHARACTERIZATION AND GEOGRAPHIC PROVENANCE OF FOOD PRODUCTS.In the past few years, the use of stable isotopes as tracers of the geographic provenance of food products is growing and the strenght of this tool in the determination of origin of products with Controlled Designation of Origin has been demonstrated. In this context, C, O, N, H, S, and Sr isotopes has been shown to be especially useful. In this paper, the basics of this method and its main techniques are reviewed, furnishing a basis for the understanding and interpretation of this kind of data.

Keywords:. Stable isotopes, geographic provenance, Controlled Designation of Origin

\section{INTRODUÇÃO}

A utilização de isótopos estáveis como traçadores naturais de origem e processos nos mais variados setores vêm ganhando força nos últimos anos, com aplicações em campos tão distintos como a ecologia, ciência forense, agronomia, geologia e arqueologia. No campo da caracterização geográfica e autenticação de produtos alimentícios, os isótopos mais usados são os de carbono, nitrogênio, oxigênio, hidrogênio e enxofre ( $\mathrm{CNOHS}$ ), além do elemento "pesado" estrôncio (Kelly et al. 2005; Ghidini et al. 2006; Gonzalvez et al. 2009; Silva et al. 2014).

O objetivo desse artigo é descrever sucintamente a teoria e os fundamentos da técnica de isótopos estáveis, além de apresentar uma breve descrição da funcionalidade e particularidades dos elementos mais utilizados, com ênfase na sua aplicação como traçadores de origem de produtos alimentícios (caracterização geográfica).

\section{ISÓTOPOS ESTÁVEIS - CONCEITOS FUNDAMENTAIS}

Cada elemento químico é definido pelo número de prótons (Z) presente no núcleo de seu átomo. 0 número de nêutrons (N) presente dentro do núcleo pode variar, dessa forma alterando a massa atômica

doi:10.18285/geonomos.v23i1.657
(A) de determinado elemento $(A=N+Z)$. Os isótopos são definidos como átomos de um mesmo elemento químico, mas com número de nêutrons diferentes, e consequentemente, com diferentes números de massa. São expressos pelo símbolo ${ }^{A} X$, onde $\mathrm{A}=$ número de massa $\mathrm{e} X=$ símbolo do elemento químico. $\mathrm{Na}$ realidade, quase todos os elementos químicos existem como uma mistura de isótopos, na qual normalmente um isótopo mais leve (com menor massa) predomina amplamente em proporção natural (Tabela 1).

Isótopos estáveis são aqueles que ocorrem de forma comum na natureza, em contraste com os isótopos radioativos, que são instáveis e dessa forma transformam-se ao longo do tempo em elementos mais estáveis, no processo conhecido como decaimento radioativo. Os isótopos resultantes do decaimento reativo de outros elementos são conhecidos como isótopos radiogênicos. Como o processo de decaimento radioativo acontece a taxas constantes, os isótopos radiogênicos têm ampla aplicação como geocronômetros, onde são utilizadas as proporções entre isótopos filho e isótopos pai para estimar o tempo decorrido desde o princípio do decaimento. $\mathrm{O}{ }^{14} \mathrm{C}$, por exemplo, é um isótopo radioativo de carbono, amplamente utilizado em radiocronometria, com meia-vida (tempo necessário para que metade dos átomos de ${ }^{14} \mathrm{C}$ decaia, transformando-se no isótopo estável ${ }^{14} \mathrm{~N}$ ) de 5730 anos. 
Tabela 1. Abundâncias naturais relativas dos principais isótopos utilizados em proveniência geográfica de alimentos (Ghidini et al., 2006).

\begin{tabular}{ccc}
\hline Elemento & Isótopo & $\begin{array}{c}\text { Abundância natural } \\
\text { relativa (\%) }\end{array}$ \\
\hline Hidrogênio & ${ }^{1} \mathrm{H}$ & 99,985 \\
& ${ }^{2} \mathrm{H}$ & 0,015 \\
\hline Carbono & ${ }^{12} \mathrm{C}$ & 98,89 \\
& ${ }^{13} \mathrm{C}$ & 1,11 \\
\hline Nitrogênio & ${ }^{14} \mathrm{~N}$ & 99,63 \\
& ${ }^{15} \mathrm{~N}$ & 0,37 \\
\hline Oxigênio & ${ }^{16} \mathrm{O}$ & 99,76 \\
& ${ }^{17} \mathrm{O}$ & 0,04 \\
& ${ }^{18} \mathrm{O}$ & 0,20 \\
\hline Enxofre & ${ }^{32} \mathrm{~S}$ & 95,00 \\
& ${ }^{33} \mathrm{~S}$ & 0,76 \\
& ${ }^{34} \mathrm{~S}$ & 4,22 \\
& ${ }^{36} \mathrm{~S}$ & 0,01 \\
\hline Estrôncio & ${ }^{84} \mathrm{Sr}$ & 0,56 \\
& ${ }^{86} \mathrm{Sr}$ & 9,86 \\
& ${ }^{87} \mathrm{Sr}$ & 7,02 \\
& ${ }^{88} \mathrm{Sr}$ & 82,56 \\
\hline
\end{tabular}

\subsection{Razão isotópica e a notação $\delta$}

Normalmente, a quantidade relativa de um determinado isótopo em uma amostra é representada pela razão entre este isótopo e outro isótopo estável do mesmo elemento. Este último é, de praxe, o isótopo leve e mais abundante do elemento em questão, sendo as análises reportadas na forma da razão isótopo pesado / isótopo leve. Dessa forma, a ênfase das análises é na diferença relativa entre amostras analisadas sob as mesmas condições, e não no valor absoluto de cada determinação. A principal vantagem dessa abordagem é o erro analítico significativamente menor, pois medidas relativas têm menos fontes de erro do que medidas absolutas (Werner \& Brand 2001).

As razões isotópicas são normalmente representadas através da notação $\delta$, em valores de $\%$ (per mil), através da fórmula:

$$
\delta=\left[\left(R_{\text {amostra }}-R_{\text {padrão }}\right) / R_{\text {padrão }}\right] * 1000
$$

Onde $R_{\text {amostra }}=$ razão isotópica medida na amostra e $R_{\text {padrão }}=$ a mesma razão de um padrão, que geralmente é um material de referência do laboratório que por sua vez é calibrado contra um padrão internacional. Dessa forma, valores $\delta$ são reportados em comparação a um padrão internacionalmente reconhecido, que é arbitrariamente fixado em $0 \%$. A tabela 2 mostra os principais padrões utilizados e suas razões isotópicas reconhecidas internacionalmente. Isótopos de carbono, por exemplo, são normalmente referenciados contra o padrão Pee Dee Belemnite (PDB), que é o valor medido em um Belemnita do Período Cretáceo da Formação Pee Dee, na Carolina do Sul, Estados Unidos. Isótopos de oxigênio podem ser tanto referenciados ao PDB quanto ao Standard Mean Ocean Water (SMOW). A aplicação do prefixo
$\mathrm{V}$ a essas siglas (V-PDB, V-SMOW) significa que o valor utilizado para a razão isotópica é aquele determinado pela International Atomic Energy Agency (IAEA), em Viena, Áustria. Um valor de $\delta$ negativo significa que a amostra é enriquecida no isótopo leve (ou empobrecida no isótopo pesado), enquanto valores positivos de $\delta$ são observados quando a amostra é enriquecida no isótopo pesado do elemento em questão, em relação ao padrão utilizado.

Tabela 2. Padrões internacionais de razão isotópica. Segundo Werner \& Brand (2001) e referências aí citadas.

\begin{tabular}{ccc}
\multicolumn{3}{c}{ Werner \& Brand (2001) e referências aí citadas. } \\
\hline $\begin{array}{c}\text { Razão } \\
\text { isotópica }\end{array}$ & Padrão Internacional & Valor do Padrão \\
\hline $\begin{array}{c}{ }^{2} \mathrm{H} /{ }^{1} \mathrm{H} \text { ou } \\
\mathrm{D} / \mathrm{H}\end{array}$ & $\begin{array}{c}\text { Vienna Standard Mean } \\
\text { Ocean Water (VSMOW) }\end{array}$ & 0,00015575 \\
& $\begin{array}{c}\text { Vienna Pee Dee Belemnite } \\
\text { (VPDB) }\end{array}$ & 0,0111802 \\
${ }^{13} \mathrm{C} /{ }^{12} \mathrm{C}$ & Ar atmosférico (AIR) & 0,0036782 \\
${ }^{15} \mathrm{~N} /{ }^{14} \mathrm{~N}$ & $\begin{array}{c}\text { Vienna Standard Mean } \\
\text { Ocean Water (VSMOW) } \\
\text { ou }\end{array}$ & VSMOW $=0,0020052$ \\
${ }^{18} \mathrm{O} /{ }^{16} \mathrm{O}$ & $\begin{array}{c}\text { VPDB }=0,0020672 \\
\\
\end{array}$ & $\begin{array}{c}\text { Vienna Pee Dee Belemnite } \\
\text { (VPDB) }\end{array}$ \\
${ }^{34} \mathrm{~S} /{ }^{32} \mathrm{~S}$ & Troilite (VCDT) & 0,0451509 \\
\end{tabular}

\subsection{Fracionamento isotópico}

Todo o poder discriminatório da técnica de isótopos estáveis deve-se aos efeitos de fracionamento isotópico, ou seja, os processos que causam mudanças nas abundâncias relativas dos isótopos entre reagentes e produtos, em determinadas reações. Dessa forma, os processos químicos, físicos e biológicos geram razões isotópicas específicas que podem ser diagnósticas da origem, do grau de pureza e dos processos de manufatura pelos quais os produtos foram submetidos.

Os dois principais mecanismos de fracionamento isotópico são o efeito isotópico cinético e o efeito isotópico termodinâmico (Galimov 1985). O efeito cinético refere-se a diferenças nas taxas de reação de isótopos distintos. Quando um elemento ou molécula envolve-se em uma reação, o isótopo mais leve forma ligações atômicas mais fracas do que o isótopo mais pesado. Dessa forma, o isótopo leve é mais reativo, concentrando-se nos produtos da reação, enquanto o isótopo pesado concentra-se nos reagentes (Coplen et al. 2002). Exemplos clássicos são os processos físicos de evaporação e condensação, que geram produtos isotopicamente mais leves (com menor razão isotópica) do que os materiais iniciais. $O$ fracionamento isotópico é mais evidente em elementos leves, tais como o hidrogênio $\left({ }^{2} \mathrm{H} /{ }^{1} \mathrm{H}\right)$, uma vez que os seus isótopos mostram diferenças de massa proporcionalmente 
maiores do que os elementos mais pesados (p. ex. $\left.{ }^{13} \mathrm{C} /{ }^{12} \mathrm{C}\right)$.

O efeito isotópico termodinâmico reflete a mudança na energia livre do sistema quando um átomo em um composto é substituído pelo seu isótopo (Galimov 1985). Um composto que possui um isótopo mais pesado apresenta uma menor reserva de energia livre, em comparação ao mesmo composto com um isótopo mais leve. Este efeito está relacionado a diferenças nas propriedades físico-químicas das amostras analisadas, evidentes em processos onde não há quebra ou formação de ligações químicas, por exemplo, durante mudanças de fase da água (sólido - líquido - vapor). Este fracionamento isotópico ocorre como resultado das diferenças da pressão de vapor das moléculas de água, que é menor para as moléculas ${ }^{1} \mathrm{H}^{1} \mathrm{H}^{18} \mathrm{O}$ e ${ }^{2} \mathrm{H}^{1} \mathrm{H}^{16} \mathrm{O}$ do que para a molécula ${ }^{1} \mathrm{H}^{1} \mathrm{H}^{16} \mathrm{O}$.

$\mathrm{Na}$ análise de dados isotópicos aplicada à caracterização geográfica de produtos alimentícios, é importante levar em consideração todos os fatores que podem causar fracionamento nos diferentes substratos. Dessa forma, é necessário avaliar a possibilidade, além do fracionamento natural nos diferentes reservatórios (oceano, atmosfera, biosfera, etc.), da ocorrência de fracionamento isotópico nos tecidos animais, no processo de manufatura dos produtos, e até mesmo durante a amostragem (Figura 1). Em relação ao fracionamento que ocorre dentro do organismo animal, cada passo na cadeia alimentar é acompanhado por um enriquecimento nos isótopos
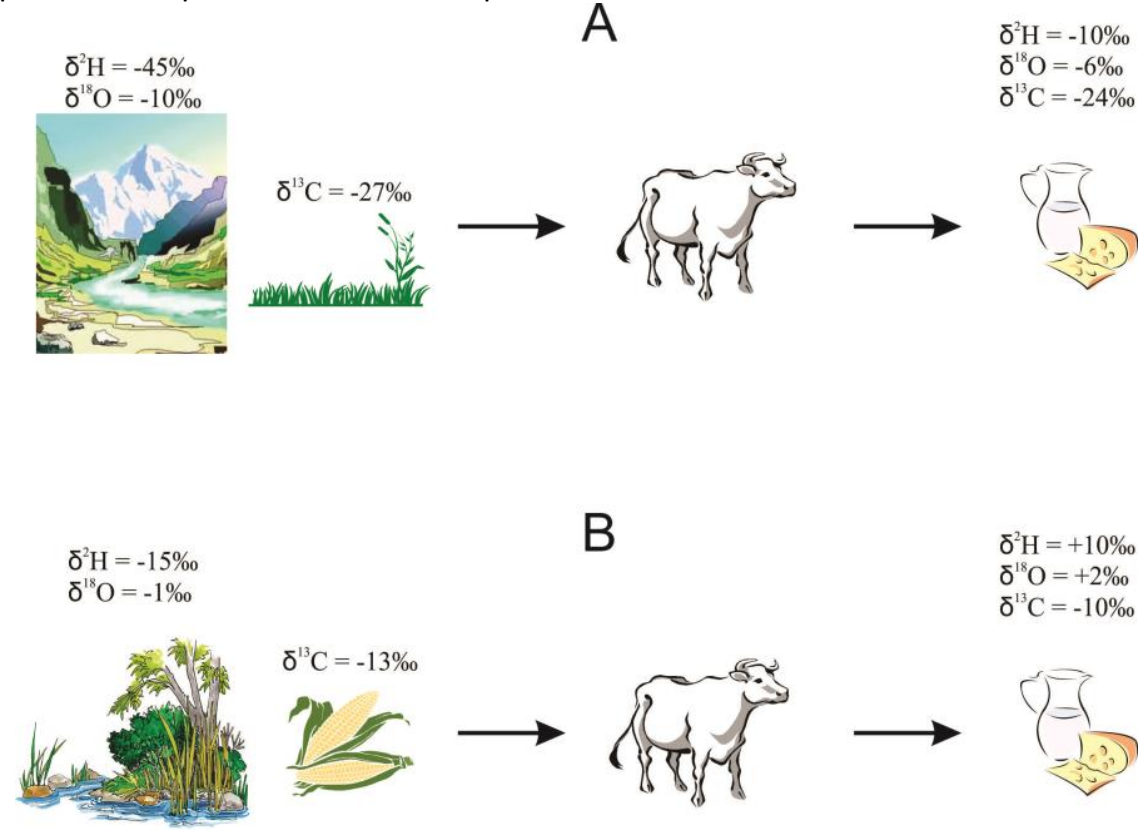

Figura 1. Ilustração da utilização de isótopos estáveis como traçadores de origem geográfica. Na situação $A$, o gado é criado em uma região montanhosa, com baixos valores de $\delta^{18} \mathrm{O}$ e $\delta^{2} \mathrm{H}$, e alimentado exclusivamente com gramíneas temperadas. Na situação $B$, o gado é criado em uma região plana e alimentado com silagem de milho. Apesar do fracionamento isotópico que ocorre no metabolismo animal e que tende a enriquecer os elementos em isótopos pesados, a análise isotópica dos produtos lácteos originados de cada animal permite reconhecer a sua origem. 


\section{ESPECTROMETRIA DE MASSA}

As razões isotópicas pertinentes para o uso na caracterização geográfica de produtos alimentícios são normalmente obtidas através de espectrômetros de massa especializados, compostos basicamente por três seções: uma fonte de íons em um extremo do aparelho, um analisador de massas em sua porção central, e um conjunto de coletores de íons no outro extremo (Figura 2). Esses equipamentos são conhecidos como espectrômetros de massa de razão isotópica (IRMS - Isotope Ratio Mass Spectrometer). A capacidade de determinar as razões isotópicas de diferentes elementos em uma mesma molécula permite a criação de uma impressão digital das moléculas utilizando mais de uma variável.

Para ser analisada, a amostra é primeiramente ionizada na fonte de íons. Normalmente são gerados íons positivos (cátions), que são então acelerados por uma diferença de potencial através de um tubo em formato curvo (tubo de voo), na porção média do qual um eletroímã gera um campo magnético que deflete as trajetórias dos íons de acordo com a razão massa / carga de cada partícula. Dessa forma, os isótopos de cada elemento são efetivamente separados em correntes iônicas distintas dentro do tubo. No outro extremo do tubo, os coletores de íons são posicionados de forma que os feixes iônicos de interesse atinjam simultaneamente cada coletor. Normalmente os coletores são do tipo copo de Faraday, onde cada partícula que atinge o fundo do copo metálico gera uma corrente elétrica; quanto maior o número de partículas, maior será a corrente gerada. As correntes são então amplificadas, digitalizadas utilizando um conversor de voltagem para frequência, e transferidas para um computador, que calcula as razões isotópicas correspondentes por comparação entre as áreas de

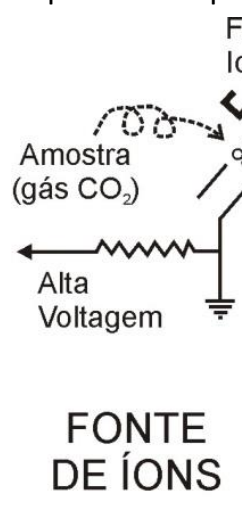

Filamento Ionizador

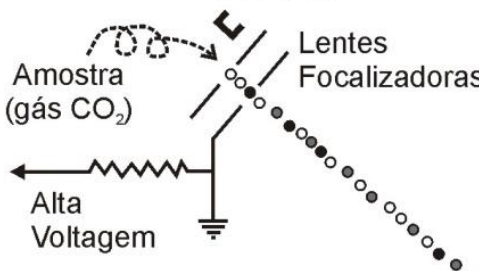

FONTE DE ÍONS pico integradas dos sinais correspondentes a cada isótopo. Por exemplo, na análise da molécula de $\mathrm{CO}_{2}$, os dados brutos consistem de três sinais correspondentes aos isotopômeros ${ }^{12} \mathrm{C}^{16} \mathrm{O}_{2},{ }^{13} \mathrm{C}^{16} \mathrm{O}_{2} \mathrm{e}$ ${ }^{12} \mathrm{C}^{18} \mathrm{O}^{16} \mathrm{O}$, separados no tubo de voo pelas suas respectivas massas 44, 45 e 46 (Figura 2). Obviamente, os íons não podem sofrer colisões com outras partículas na fonte ou no interior do tubo, o que causaria o desvio dos feixes iônicos. Caso isto ocorresse, as correntes medidas nos coletores não corresponderiam à composição isotópica original da amostra. É necessário, então, que todo o interior do aparelho funcione em ambiente de alto vácuo, o que é atingido através do uso de bombas distintas para a fonte e para o tubo.

A maioria dos isótopos úteis na caracterização geográfica de alimentos pode ser medida através de IRMS de fonte gasosa, ou seja, as amostras líquidas, sólidas ou gasosas são convertidas em gases simples antes de serem analisadas. Medidas das razões isotópicas ${ }^{2} \mathrm{H} /{ }^{1} \mathrm{H},{ }^{15} \mathrm{~N} /{ }^{14} \mathrm{~N},{ }^{13} \mathrm{C} /{ }^{12} \mathrm{C},{ }^{18} \mathrm{O} /{ }^{16} \mathrm{O}$ e ${ }^{34} \mathrm{~S} /{ }^{32} \mathrm{~S}$ são realizadas a partir dos gases de $\mathrm{H}_{2}, \mathrm{~N}_{2}, \mathrm{CO}_{2}, \mathrm{CO}$ e $\mathrm{SO}_{2}$, respectivamente. Para a determinação de razões isotópicas de elementos com baixo potencial de ionização, tais como o estrôncio e o chumbo, a análise através de espectrômetros de massa de ionização termal (TIMS - Thermal Ionisation Mass Spectrometer) é indicada. Nesses equipamentos, a amostra é carregada em formato sólido em um filamento metálico, comumente de rênio ou tungstênio altamente purificados, que é aquecido a altas temperaturas na fonte de íons, até que a amostra seja ionizada. Alternativamente às análises por TIMS, as amostras podem ser analisadas em um espectrômetro de massa de plasma induzido (ICPMS - Induced Coupled Plasma Mass Spectrometer). Neste equipamento, as amostras são introduzidas em solução e borrifadas em um plasma de argônio a altas temperaturas, onde os íons são gerados.

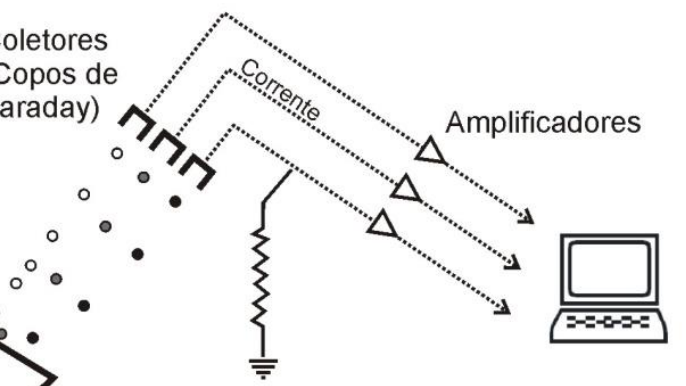

\section{COLETORES}

$$
\text { - } 46\left({ }^{12} \mathrm{C}^{18} \mathrm{O}^{16} \mathrm{O}\right) \quad \text { ○ } 45\left({ }^{13} \mathrm{C}^{16} \mathrm{O}_{2}\right) \quad 044\left({ }^{12} \mathrm{C}^{16} \mathrm{O}_{2}\right)
$$

Figura 2. Esquema de funcionamento de um espectrômetro de massa de razão isotópica (IRMS). No exemplo, uma amostra de CO ${ }_{2}$ é analisada e as moléculas de diferentes massas (44, 45 e 46), de acordo com os isótopos de C e O envolvidos, são defletidas pelo campo magnético do eletroímã e coletadas pelos copos de Faraday. 
O principal problema das análises por IRMS são as interferências isobáricas de outros elementos e compostos que podem apresentar massa semelhante ao isótopo em questão. Dessa forma, a separação química dos elementos desejados é necessária antes da sua análise no espectrômetro de massa. Essa separação deve ser feita em ambiente de sala limpa, com pressão positiva de ar, para evitar a contaminação da amostra nas diversas etapas do processo. No caso do estrôncio, por exemplo, quatro isótopos são medidos $\left({ }^{84} \mathrm{Sr},{ }^{86} \mathrm{Sr},{ }^{87} \mathrm{Sr}\right.$ e $\left.{ }^{88} \mathrm{Sr}\right)$, e as principais interferências isobáricas são ${ }^{87} \mathrm{Rb}$, ${ }^{44} \mathrm{Ca}^{40} \mathrm{Ar},{ }^{48} \mathrm{Ca}^{40} \mathrm{Ar}$ e elementos terras raras com carga +2 (p. ex. ${ }^{167} \mathrm{Er}^{2+},{ }^{168} \mathrm{Yb}^{2+}$ ) provenientes da amostra, além de ${ }^{84} \mathrm{Kr}$ e ${ }^{86} \mathrm{Kr}$ provenientes do gás de argônio utilizado na operação do equipamento no caso do ICPMS (Fortunato et al. 2004). Uma separação química eficiente, através de cromatografia em micro-colunas utilizando resinas para a recuperação do elemento desejado, aliada a procedimentos computadorizados de correção durante a análise, geralmente permite contornar estes problemas e atingir medidas altamente precisas e acuradas.

O acoplamento de um equipamento de ablação a laser (Laser Ablation) a um espectrômetro de massa do tipo ICP abre ainda a possibilidade de analisar amostras sólidas in situ (LA-ICPMS), em contraste com as outras técnicas, que exigem a dissolução da amostra e a separação química dos elementos de interesse antes da análise.

\section{APLICAÇÕES}

\subsection{Isótopos de C}

O carbono possui dois isótopos estáveis, ${ }^{13} \mathrm{C} \mathrm{e}{ }^{12} \mathrm{C}$ (Tabela 1). De especial interesse na caracterização geográfica de alimentos é a incorporação seletiva de ${ }^{12} \mathrm{C}$ na matéria orgânica durante $\mathrm{o}$ processo de fotossíntese, decorrente do efeito cinético de fracionamento isotópico. $\mathrm{O} \mathrm{CO}_{2}$ atmosférico $\left(\delta^{13} \mathrm{C}\right.$ ao redor de $-8 \%$ ) assimilado durante a fotossíntese sofre fracionamento isotópico considerável no organismo das plantas. O principal fator que controla o grau de fracionamento é o ciclo fotossintético específico utilizado pela planta para a fixação do carbono, embora outros fatores metabólicos e ambientais também influenciem na assinatura isotópica da planta (p. ex. umidade, temperatura, composição isotópica do solo).

As plantas assimilam $\mathrm{CO}_{2}$ através dos ciclos fotossintéticos $\mathrm{C}_{3}$ (Calvin), $\mathrm{C}_{4}$ (Hatch-Slack) ou CAM (Crassulacean Acid Metabolism). A maioria das plantas segue o ciclo fotossintético $C_{3}$, incluindo importantes culturas tais como arroz, cevada, trigo, soja, leguminosas, hortaliças e frutíferas em geral, além de gramíneas temperadas (Ducatti et al., 2011). Por outro lado, como exemplos importantes de plantas $\mathrm{C}_{4}$ podem ser citados a cana de açúcar, o milho e as gramíneas tropicais. As plantas $C_{3}$ incorporam preferencialmente $0{ }^{12} \mathrm{C}$ porque reduzem $\mathrm{O} \mathrm{CO}_{2}$ a fosfoglicerato através da enzima RuBP-carboxilase, que é sensível às diferenças de massa dos isótopos de carbono; já as plantas $\mathrm{C}_{4}$ reduzem $\circ \mathrm{CO}_{2}$ a ácido aspártico ou málico através da enzima PEP-carboxilase, que não possui o poder discriminatório da RuBP-carboxilase (Boutton 1991; Oliveira et al., 2002). Dessa forma, os valores de $\delta^{13} \mathrm{C}$ das plantas $\mathrm{C}_{3}$, que assimilam preferencialmente $\mathrm{O}$ ${ }^{12} \mathrm{C}$, varia entre -22 e $-34 \%$, enquanto nas plantas $C_{4}$ o $\delta^{13} \mathrm{C}$ varia entre -9 a $-16 \%$ (Vogel 1993; Boutton 1991, 1996; Ducatti et al. 2011). Esses valores isotópicos agrupados em intervalos distintos e sem sobreposição permitem identificar a dieta dos animais a partir da análise isotópica dos produtos em questão, uma vez que a assinatura isotópica da fonte alimentar é refletida nos tecidos específicos dos animais após certo tempo de metabolismo (DeNiro \& Epstein 1978; Gannes et al. 1998; Ducatti et al. 2011), e por sua vez essa assinatura é transmitida aos produtos de origem animal tais como os laticínios (Figura 1).

Uma vez que a dieta reflete fatores climáticos e geográficos, os isótopos de carbono podem ser utilizados para a caracterização geográfica de produtos animais. Em regiões de clima temperado, por exemplo, predominam as gramíneas $C_{3}$, e a adição de quantidades variáveis de plantas $C_{4}$ na dieta dos animais, tais como a silagem de milho, é rapidamente identificada através do desvio dos valores isotópicos de $\delta^{13} \mathrm{C}$ em direção a valores menos negativos. Misturas entre plantas $C_{3}$ e $C_{4}$ na dieta produzem valores intermediários de $\delta^{13} \mathrm{C}$, entre -16 e $-22 \%$. Essa variação pode também refletir efeitos sazonais, uma vez que durante o inverno, quando a quantidade de gramíneas nas regiões temperadas é menos abundante do que no verão, os produtores podem misturar maiores quantidades de silagem de milho na dieta dos animais, quando esta prática é permitida por lei. $\mathrm{Na}$ maior parte do Brasil, predominam as gramíneas tropicais do tipo $\mathrm{C}_{4}$.

\subsection{Isótopos de $\mathbf{N}$}

De forma distinta ao carbono, a composição isotópica do nitrogênio nas plantas não depende do ciclo fotossintético utilizado, e sim do modo de fixação deste elemento. Somente as plantas que possuem associação com bactérias fixadoras de nitrogênio conseguem assimilá-lo a partir da atmosfera $\left(\mathrm{N}_{2}\right)$, enquanto a maioria das plantas fixa o nitrogênio a partir da assimilação de compostos nitrogenados do solo. Dessa forma, o principal fator controlador da composição isotópica do nitrogênio nas plantas é a assinatura isotópica original do solo, que por sua vez é influenciada por fatores diversos 
tais como as práticas de agricultura (extensiva $\mathrm{x}$ intensiva), e as condições climáticas e geográficas predominantes.

A utilização de grandes quantidades de fertilizante orgânico tende a aumentar significativamente $\mathrm{o}$ valor de $\delta^{15} \mathrm{~N}$ nos solos, atingindo valores em torno de $10 \%$ (Shibuya et al. 2006). Fatores dependentes das condições geográficas, tais como a aridez, salinidade e proximidade do mar, também tendem a aumentar o valor de $\delta^{15} \mathrm{~N}$. De modo contrário, valores negativos de $\delta^{15} \mathrm{~N}$ são observados em solos com baixa porcentagem de matéria orgânica, fertilizados com adubos industriais ou em solos secos e arenosos (Ducatti et al. 2011). Os adubos industriais possuem valores de $\delta^{15} \mathrm{~N}$ em torno de $0 \%$, por utilizar nitrogênio atmosférico em sua fabricação, dessa forma aproximando-se do padrão AIR (composição isotópica do nitrogênio atmosférico na superfície terrestre, considerada como uma mistura homogênea). Da mesma forma, a adição de plantas com microorganismos fixadores de nitrogênio atmosférico na dieta animal também tende a reduzir o valor de $\delta^{15} \mathrm{~N}$ nos tecidos e produtos animais. Por exemplo, a maioria das leguminosas realiza simbiose com micorrizas, que fixam o nitrogênio atmosférico e conduzem a assinatura isotópica dessas plantas para valores de $\delta^{15} \mathrm{~N}$ em torno de $0 \%$ (Handley \& Raven 1992; Werner \& Schimidt 2002; Ducatti et al. 2011).

\subsection{Isótopos de $\mathrm{O}$ e $\mathrm{H}$}

Os valores de $\delta^{18} \mathrm{O}$ e de $\delta^{2} \mathrm{H}$ (ou $\delta \mathrm{D}=$ deutério) nos produtos alimentícios dependem da composição isotópica da água $\left(\mathrm{H}_{2} \mathrm{O}\right)$ ingerida pelos animais, que por sua vez é influenciada primordialmente por fatores geográficos tais como altitude, latitude e distância do mar (Moser \& Rauert 1980). A proporção entre forragem fresca e forragem seca na dieta, além de efeitos sazonais, também podem ser fatores importantes. No verão, por exemplo, devido às altas taxas de evapotranspiração, as plantas apresentam valores enriquecidos de $\delta^{18} \mathrm{O}$ (enriquecidos no isótopo pesado, já que o isótopo leve é preferencialmente incorporado nas moléculas evaporadas).

O principal fator que controla a composição isotópica do $\delta^{18} \mathrm{O}$ é a evaporação da água, influenciada pelas condições climáticas tais como temperatura, umidade e pluviosidade (Simpkins et al. 1999). Dessa forma, plantas em clima quente e seco apresentam valores mais enriquecidos de $\delta^{18} \mathrm{O}$ do que aquelas em ambientes de clima úmido e fresco. Por exemplo, Crittenden et al. (2007) demonstram que amostras de leite da Australásia apresentam valores de $\delta^{18} \mathrm{O}$ entre +3 e $-2 \%$, em comparação com amostras de leite da Europa central e setentrional que apresentam valores de $\delta^{18} \mathrm{O}$ bem mais empobrecidos, entre -2 e $-11 \%$ 。 (Kornexl et al. 1997; Rossmann et al. 1998; Pillonel et al. 2003; Renou et al. 2004), refletindo as diferenças climáticas entre essas duas regiões.

A altitude é também um fator muito importante na composição isotópica da água. Em áreas montanhosas, a água tende a ser enriquecida nos isótopos leves, dessa forma com valores mais negativos de $\delta^{2} \mathrm{H}$ e $\delta^{18} \mathrm{O}$ do que em áreas planas e de cotas mais baixas (Renou et al. 2004). A proporção entre o deutério e o hidrogênio em moléculas de água segue um padrão análogo ao oxigênio na hidrosfera.

\subsection{Isótopos de $S$}

Os fatores que afetam a composição isótopica do enxofre nos produtos animais são complexos e ainda não são completamente compreendidos, o que dificulta na interpretação dos dados de $\delta^{34} \mathrm{~S}$. Sabe-se que o substrato geológico é o fator mais importante na determinação da composição isotópica do enxofre no solo, com diferenças significativas entre terrenos de diferentes naturezas, p. ex. escudos cristalinos compostos predominantemente por rochas ígneas versus áreas de bacia sedimentar; ou áreas de rochas predominantemente ácidas (ricas em sílica) versus áreas de rochas básicas (pobres em sílica; Rossman et al. 1998). Outros fatores, tanto relacionados às práticas agrícolas quanto ao clima, também são importantes, tais como os procedimentos de fertilização utilizados e a proximidade com o oceano, já que a água marinha é enriquecida em $\delta^{34} S(\sim 22 \%$ ) em relação aos sulfatos do solo (Chivas et al. 1991; Rossmann 2001). Emissões ricas em $\mathrm{SO}_{2}$ também podem influenciar a composição isotópica do enxofre nos solos e insumos.

O padrão utilizado internacionalmente para comparação da razão isotópica ${ }^{34} \mathrm{~S} /{ }^{32} \mathrm{~S}$ é o Canyon Diablo Troilite (CDT), que é uma variedade de pirrotita (sulfeto de ferro, FeS) encontrada em um fragmento do meteorito que impactou a Terra há aproximadamente 50.000 anos atrás, criando a Cratera do Meteoro, no Arizona, Estados Unidos.

\subsection{Isótopos de $\mathrm{Sr}$}

O estrôncio é um metal alcalino terroso do grupo IIA que possui quatro isótopos de ocorrência natural, ${ }^{84} \mathrm{Sr},{ }^{86} \mathrm{Sr},{ }^{87} \mathrm{Sr}$ e ${ }^{88} \mathrm{Sr}$. $\quad{ }^{87} \mathrm{Sr}$ é particularmente importante, por derivar do decaimento radioativo do tipo $\beta$ do ${ }^{87} \mathrm{Rb}$, com meia-vida de 49 bilhões de anos (Faure 1986). A abundância de ${ }^{87} \mathrm{Sr}$ em uma determinada amostra é a soma de duas fontes: 0 ${ }^{87} \mathrm{Sr}$ inicial, formado pela nucleossíntese primordial conjuntamente aos outros isótopos estáveis de $\mathrm{Sr}$, e $0{ }^{87} \mathrm{Sr}$ advindo do decaimento radioativo de ${ }^{87} \mathrm{Rb}$. 
Dessa forma, a concentração de ${ }^{87} \mathrm{Sr}$ em uma rocha depende das concentrações iniciais de $\mathrm{Rb}$ e $\mathrm{Sr}$ e da idade da rocha; quanto maior a razão $\mathrm{Rb} / \mathrm{Sr}$ e a idade, maior será a razão ${ }^{87} \mathrm{Sr} /{ }^{86} \mathrm{Sr}$ e vice-versa. Esse efeito é refletido na diferença isotópica entre terrenos antigos e / ou dominados por rochas cristalinas, graníticas, que possuem altas razões iniciais $\mathrm{Rb} / \mathrm{Sr}$ e, por conseguinte, altos valores de ${ }^{87} \mathrm{Sr} /{ }^{86} \mathrm{Sr}$, tipicamente acima de 0.710 , enquanto em terrenos dominados por rochas basálticas ou rochas mais jovens, as razões ${ }^{87} \mathrm{Sr} /{ }^{86} \mathrm{Sr}$ se apresentam tipicamente entre 0.702 e 0.705 .

Em contraste com os outros isótopos utilizados para a caracterização geográfica de alimentos, processos biológicos, climáticos ou sazonais não causam fracionamento isotópico significativo nos isótopos de estrôncio, e portanto a razão ${ }^{87} \mathrm{Sr} /{ }^{86} \mathrm{Sr}$ é efetivamente herdada do substrato geológico, embora alguns fatores locais, tais como diferenças na taxa de intemperismo de minerais distintos, aplicação de fertilizantes e precipitação de águas marinhas possam influenciar as biodisponibilidade de estrôncio no solo (Martin \& McCulloch 1999). A razão isotópica ${ }^{87} \mathrm{Sr} /{ }^{86} \mathrm{Sr}$ é, dessa forma, uma poderosa ferramenta de indicação de origem geográfica de uma amostra, contanto que a composição isotópica do substrato geológico e que a possível presença de litologias de idade e composição distintas sejam conhecidas para a região em apreço.

De forma semelhante aos outros isótopos, um valor de $\delta^{87} \mathrm{Sr}$ é por vezes utilizado ao invés das razões absolutas (p. ex., Rossmann et al. 2000), calculado através da fórmula: $\delta^{87} \mathrm{Sr}=$ $\left[\left({ }^{87} \mathrm{Sr} /{ }^{86} \mathrm{Sr}_{\text {amostra }}\right) / 0.7093\right]-1 \times 1000$. Neste caso, a razão 0.7093 é assumida como um valor padrão de ${ }^{87} \mathrm{Sr} /{ }^{86} \mathrm{Sr}$ e o valor de $\delta^{87} \mathrm{Sr}$ indica o desvio dos valores medidos em relação a esse valor padrão, em per mil (\%o).

\section{CONCLUSÕES}

A utilização de isótopos estáveis como traçadores é uma poderosa ferramenta na identificação da origem e pureza de produtos animais, especialmente quando as razões isotópicas de mais de um elemento são medidas nas mesmas amostras, dessa forma produzindo múltiplas variáveis atreladas à origem e aos processos pelos quais os produtos foram submetidos.

Dentre os elementos que mais são utilizados na caracterização geográfica de alimentos, os isótopos de carbono refletem principalmente a dieta dos animais; os isótopos de nitrogênio refletem as práticas agrícolas (extensiva $\mathrm{x}$ intensiva) e em parte a dieta; os isótopos de oxigênio e hidrogênio são vinculados à composição isotópica da água que, por sua vez, é dependente de fatores geográficos tais como altitude, clima e latitude; os dados isotópicos de enxofre dependem de vários fatores e são mais difíceis de interpretar, embora o principal fator controlador seja o substrato geológico, além de fatores geográficos e antrópicos; e os isótopos de estrôncio são indicadores efetivos de proveniência geográfica, uma vez que estão diretamente atrelados à razão isotópica do substrato geológico e não passam por fracionamento significativo através de processos biológicos.

\section{REFERÊNCIAS BIBLIOGRÁFICAS}

Boutton, T.W. 1991. Stable carbon isotope ratios of natural materials: II. Atmospheric, terrestrial, marine, and freshwater environments. In: Coleman, D.C., Fry, B. (eds). Carbon Isotope Techniques. New York: Academic Press, p. 173-185.

Boutton, T.W. 1996. Stable carbon isotope ratios of soil organic matter and their use as indicators of vegetation and climate change. In: Boutton, T.W.; Yamasaki, S-I. (Eds.) Mass spectrometry of soils. New York: Marcel Dekker, p.47-82.

Chesson, L.A., Valenzuela, L.O., O’Grady, S.P., Cerling, T.E., Ehleringer, J.R., 2010. Hydrogen and oxygen stable isotope ratios of milk in the United States. Journal of Agricultural and Food Chemistry, 58(4): 2358-2363.

Chivas, A.R.; Andrew, A.S.; Lyons, W.B.; Bird, M.I.; Donnelly, T.H. 1991. Isotopic constraints on the origin of salts in Australian playas. I. Sulphur. Palaeogeography, Palaeoclimatology, Palaeoecology 84: 309-332.

Coplen, T.B.; Bohlke, J.K.; De Bievre, P.; Ding, T.; Holden, N.E.; Hopple, J.A.; Krouse, H.R.; Lamberty, A.; Peiser, H.S.; Revesz, K.; Rieder, S.E.; Rosman, K.J.R.; Roth, E.; Taylor, P.D.P.; Vocke Jr., R.D.; Xiao, Y.K. 2002. Isotope-abundance variations of selected elements (IUPAC Technical Report), Pure and Applied Chemistry 74(10): 1987-2017

Crittenden, R.G.; Andrew, A.S.; Lefournour, M.; Young, M.D.; Middleton, H.; Stockmann, R. 2007. Determining the geographic origin of milk in Australasia using multi-element stable isotope ratio analysis. International Dairy Journal, 17:421-428.

DeNiro, M.J. \& Epstein, S. 1978. Influence of diet on the distribution of carbon isotopes in animals. Geochimica et Cosmochimica Acta, 42:495-506.

Ducatti, C.; Martins, C.L.; Arrigoni, M.B.; Martins, M.B.; Vieira Jr., L.C.; Denadai, J.C. 2011. Utilização de isótopos estáveis em ruminantes. R. Bras. Zootec., v.40, p.68-75, 2011 (supl. especial)

Faure, G. 1986. Principles of isotope geology, $2^{\text {nd }}$ ed. New York: Wiley.

Fortunato, G.; Mumic, K.; Wunderli, S.; Pillonel, L.; Bosset J.O.; Gremaud G. 2004. Application of strontium isotope abundance ratios measured by MC-ICP-MS for food authentication. Journal of Analytical Atomic Spectrometry 19:227-234

Galimov, E.M. 1985. The Biological Fractionation of Isotopes, Academic Press, Florida.

Gannes, L.Z.; Del-Rio, C.M.; Koch, P. 1998. Natural abundance variations in stable isotopes and their potential uses in animal physiological ecology. Comparative Biochemistry Physiology, 119:725-737.

Ghidini, S.; Ianieri, A.; Zanardi, E.; Conter, M.; Boschetti, T.; Iacumin, P.; Bracchi, P.G. 2006. Stable isotopes determination 
in food authentication: a review. Ann. Fac. Medic. Vet. di Parma 26: 193-204.

Gonzalvez, A.; Armenta, S.; De la Guardia, M. 2009. Trace-element composition and stable-isotope ratio for discrimination of foods with Protected Designation of Origin. Trends in Analytical Chemistry, 28(11):1295-1311.

Handley, L.L. \& Raven, J.A. 1992. The use of natural abundance of nitrogen isotopes in plant physiology and ecology. Plant Cell and Environment, 15:965-985.

Kelly, S.; Heaton, K.; Hoogewerff, J. 2005. Tracing the geographical origin of food: The application of multi-element and multiisotope analysis. Trends in Food Science \& Technology 16: 555-567.

Kornexl, B.E.; Werner, R.; Rossmann, A.; Schmidt, H.-L. 1997. Measurement of stable isotope abundances in milk and milk ingredients - A possible tool for origin assignment and quality control. Zeitschrift fur Lebensmittel-Untersuchung undForschung A, 205(1):19-24.

Kroopnick, P.; Craig, H. 1972. Atmospheric oxygen: isotopic composition and solubility fractionation. Science, 175, 54-55.

Martin, C.E. \& McCulloch, M.T. 1999. Nd-Sr isotopic and trace element geochemistry of river sediments and soils in a fertilized catchment, New South Wales, Australia. Geochimica et Cosmochimica Acta, 63:287-305.

Metges, C.; Kempe, K.; Schmidt, H-L 1990. Dependence of the carbon-isotope contents of breath carbon dioxide, milk, serum and rumen fermentation products on the $\delta 13 \mathrm{C}$ value of food in dairy cows. British Journal of Nutrition 63: 187-196.

Moser, H. \& Rauert, W. 1980. Isotopenmethoden in der Hydrologie. Berlim: Borntraeger, 400 p.

Nakamura, K.; Schoeller, D.A.; Winkler F.J.; Schmidt, H-L. 1982. Geographical variations in the carbon isotope composition of the diet and hair in contemporary man. Biological Mass Spectrometry 9:390-394

Oliveira, A.C.B.; Salimon, C.I.; Calheiros, D.F.; Fernandes, F.A.; Vieira, I.; Charbel, L.F.; Pires, L.F.; Salomão, M.S.M.B.; Nogueira, S.F.; Vieira, S. 2004. Isótopos estáveis e produção de bebidas: de onde vem o carbono que consumimos? Ciência e Tecnologia de Alimentos, 22(3):285-288.

Pillonel, L.; Badertscher, R.; Froidevaux, P.; Haberhauer, G.; Holzl, S.; Horn, P.; Jakob, A.; Pfammatter, E.; Piantini, U.; Rossmann, A.; Tabacchi, R.; Bosset, J.O. 2003. Stable isotope ratios, major, trace and radioactive elements in emmental cheeses of different origins. Lebensmittel-Wissenschaft undTechnologie 36(6): 615-623.

Renou, J.P.; Deponge, C.; Gachon, P.; Bonnefoy, J.C.; Coulon, J.B.; Garel, J.P., Vérité, R.; Ritz, P. 2004. Characterization of animal products according to geographic origin and feeding diet using nuclear magnetic resonance and isotope ratio mass spectrometry: Cow milk. Food Chemistry, 85(1): 63-66.
Ritz, P.; Cole, T. J.; Davies, P. S. W.; Goldeberg, G. R.; Coward, W. A, 1996. Interactions between $2 \mathrm{H}$ and 180 natural abundance variations and DLW measurements of energy expenditure. Am. J. Physiol., 271, 302-308.

Ritz, P.; Gachon, P.; Garel, J.P.; Bonnefoy, J.C.; Coulon, J.B.; Renou, J.P. 2005. Milk characterization: Effect of the breed. Food Chemistry, 91:521-523.

Roden, J.; Lin, G.; Ehleringer, J., 2000. A mechanistic model for interpretation of hydrogen and oxygen isotope ratios in treering cellulose. Geochim. Cosmochim. Acta, 64, 21-35.

Rossmann, A.; Kornexl, B.E.; Versini, G.; Pichlmayer, F.; Lamprecht, G. 1998. Origin assignement of milk from alpine regions by multielement stable isotope ratio analysis (SIRA). Journal of Food Science and Nutrition, 1:9-21.

Rossmann, A. 2001. Determination of stable isotope ratios in food analysis. Food Reviews International, 17(3):347-381.

Rossmann, A.; Haberhauer, G.; Holzl, S.; Horn, P.; Pichlmayer, F. Voerkelius, S. (2000). The potential of multielement stable isotope analysis for regional origin assignment of butter. European Food Research and Technology, 211(1): 32-40.

Schmidt, H.-L.; Werner, R.; Rossmann, A, 2001. 180 Pattern and biosynthesis of natural plant products. Phytochemistry 58:93.

Schoeninger, M.J.; DeNiro, M.J.; Tauber, H. 1983. Stable nitrogen isotope ratios of bone collagen reflect marine and terrestrial components of prehistoric human diet. Science 220:13811383

Shibuya, E.K.; Sarkis, J.E.S.; Negrini Neto, O.; Moreira, M.Z.; Victoria, R.L. 2006. Sourcing Brazilian marijuana by applying IRMS analysis to seized samples. Forensic Science International, 160(1):35-43.

Silva, A.V.; Hélie, J.F.; Caxito, F.A.; Monardes, H.; Mustafa, A.F. Stevenson, R. 2014. Multi-stable isotope analysis as a tool for assessing the geographic provenance of dairy products: A case study using buffalo's milk and cheese samples from the Amazon basin, Brazil. International Dairy Journal, 35:107-110. Doi: 10.1016/j.idairyj.2013.10.019

Simpkins, W.A.; Patel, G.; Collins, P.; Harrison, M.; Goldberg, D. 1999. Oxygen isotope ratios of juice water in Australian oranges and concentrates. Journal of Agriculture and Food Chemistry, 47:2606-2612.

Vogel, J.C. 1993. Variability of carbon isotope fractionation during photosynthesis. In: Ehleringer, J.R.; Hall, A.E.; Farquhar, G.D. (Eds.). Stable isotopes and plant carbon: water relations. San Diego: Academic Press, p.29-46.

Werner, R.A. \& Brand, W.A. 2001. Referencing strategies and techniques in stable isotope ratio analysis. Rapid Communications in Mass Spectrometry, 15:501-519.

Werner, R.A. \& Schimidt, H.-L. 2002. The in vivo nitrogen isotope discrimination among organic plant compounds. Phytochemistry, 61: p.465-784. 